\title{
Blue Liquid Lasers from Solution of CdZnS/ZnS Ternary Alloy Quantum Dots with Quasi-Continuous Pumping
}

\author{
Yue Wang, Kheng Swee Leck, Van Duong Ta, Rui Chen, Venkatram Nalla, Yuan Gao, \\ Tingchao He, Hilmi Volkan Demir,* and Handong Sun*
}

\begin{abstract}
Photonic devices based on semiconductor colloidal quantum dots (QDs) have attracted a great deal of attention due to the facile solution-processability, wide range spectral tunability, and potentially low and temperature-insensitive lasing threshold..$^{[1,2]}$ Although white-light-emitting diodes based on colloidal QDs have been demonstrated, ${ }^{[3,4]}$ fabricating colloidal QD lasers across the full visible range is still challenging, hindered by the realization of blue QD lasers. ${ }^{[1,5]}$ On the other hand, blue lasers hold important applications ranging from the development of full-color laser displays to high-density photomemories. ${ }^{\left[{ }^{6]}\right.} \mathrm{How}-$ ever, generally the overwhelmingly employed CdSe-based colloidal QDs show lasing only in the green to red regime with wavelengths longer than $500 \mathrm{~nm} \cdot .^{[1,7]}$ Extending the lasing wavelength down to the blue range requires further reduction of the dot size for stronger quantum confinement, thereby rendering the higher nonradiative Auger recombination (AR) rate and more efficient excited carriers trapping in small-sized blueemitting QDs. ${ }^{[1,7,8]}$ Furthermore, most of the lasing demonstrations are limited in the form of solid films by taking advantage of the available high packing-density exceeding the critical value predicted by Klimov et al. ${ }^{[1,2,5,7,9-11]}$ Whilst, lasing from a liquid medium favors the development of high power lasers for the sake of the facilities of heat dissipation ${ }^{[12]}$ and the development of optofluidic lasers ${ }^{[13,14]}$ which have great potential in biological research. However, only a limited number of studies have demonstrated lasing action from QDs in solution phase, ${ }^{[15,16]}$
\end{abstract}

\footnotetext{
Y. Wang, V. D. Ta, Dr. R. Chen, Y. Gao,

Dr. T. C. He, Prof. H. D. Sun

Division of Physics and Applied Physics

School of Physical and Mathematical Sciences

Nanyang Technological University

Singapore 637371, Singapore

E-mail: HDSun@ntu.edu.sg

K. S. Leck, Prof. H. V. Demir

School of Electrical and Electronic Engineering

Nanyang Technological University

Nanyang Avenue

Singapore 639798, Singapore

E-mail: HVDemir@ntu.edu.sg

Dr. V. Nalla, Prof. H. V. Demir, Prof. H. D. Sun

Centre for Disruptive Photonic Technologies (CDPT)

Nanyang Technological University

Singapore 637371, Singapore

Prof. H. V. Demir

Department of Electrical and Electronics Engineering

Department of Physics, UNAM - Institute of Materials

Science and Nanotechnology

Ankara, Turkey 06800
}

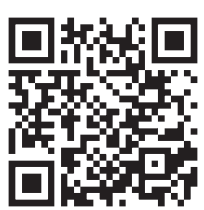

DOI: 10.1002/adma.201403237 and all of these demonstrations are restricted in red spectral region. ${ }^{[15,16]}$ Recently, Nurmikko et al. have shown blue lasing from self-assembled films of unconventional pyramid-shaped CdSe/ZnCdS QDs but with threshold ten-times higher than that of the red counterpart, ${ }^{[9]}$ which might necessitate to employ costly femtosecond lasers as the pumping source. Herein, we demonstrate blue lasing $(\sim 40 \mathrm{~nm})$ in solution with such a low threshold that quasi-continuous wave (q-CW) pumping is accessible by engineering high quality $\mathrm{CdZnS/ZnS}$ alloyed-core/ shell QDs (alternative to the conventional CdSe-based QDs) as gain media. ${ }^{[17,18]}$ Detailed lasing characterization and analysis attribute the longitudinal laser modes to whispering gallery mode (WGM) lasing. The physical mechanism for the achievement of blue lasing from CdZnS/ZnS QDs solution with ultralow thresholds has been investigated in detail through steady state and ultrafast dynamics studies, and is attributed to the low density of defects, suppressed AR, large gain cross-section of $\mathrm{CdZnS} / \mathrm{ZnS} \mathrm{QDs}$ and the exploiting of high quality factor ( $Q$-factor) WGM resonators. Our results represent an important step toward the development of full-color visible lasers based on facile colloidal QDs and may revitalize optofluidic lasers in which fluorescent dyes with poor photostability are usually used as gain media ${ }^{[6,9,14]}$ as well as offering new possibilities in biophotonics, such as laser-assisted sensing since the blue lasing wavelength falls in the absorption band of most biomolecules. ${ }^{[19]}$

Leveraging on the wide band gap of $\mathrm{ZnS}$ and $\mathrm{CdS}$, the ternary CdZnS/ZnS QDs could exhibit blue emission even with a particle radius close to or larger than the corresponding exciton Bohr radius, where the nonradiative $A R$ is not efficient. ${ }^{[7]}$ Notably, the radially graded alloy structure (schematically portrayed in Figure 1a inset) is engineered due to the potential reduction of interface defects and suppression of $A R,{ }^{[20,21]}$ as elaborated below. Therefore, the obstacles to achieve blue lasing for CdSe-based QDs could be effectively overcome by using the CdZnS/ZnS alloyed-core/shell QDs. The CdZnS/ZnS QDs adopted here are produced through the previously reported facile one-pot method with slight modification (see Experimental Section). ${ }^{[20,22]}$ By simply adjusting the quantity of sulfur precursors, the emission wavelengths of these QDs can be continuously tuned from $\sim 410$ to $\sim 460 \mathrm{~nm}$ maintaining excellent optical properties including the strong band edge emission with narrow full-width at half maximum (FWHM) and high quantum yields. ${ }^{[20]}$ Figure 1a shows the representative steady state linear absorption and photoluminescence (PL) spectra with emission peak wavelength of $435 \mathrm{~nm}$. The extreme narrow FWHM of $\sim 18 \mathrm{~nm}$ at room temperature is mainly attributed to the high composition homogeneity, the narrow size distribution and the large particle size $(\sim 8.5 \mathrm{~nm})$ of these CdZnS/ZnS QDs, 

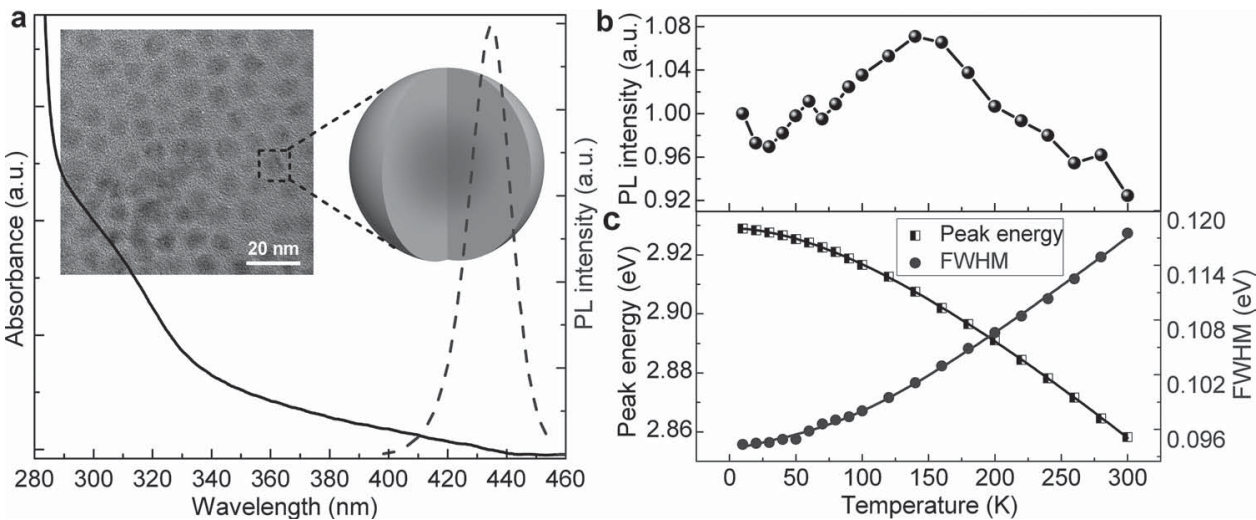

Figure 1. a) Absorption and emission spectra $(F W H M=18 \mathrm{~nm})$ of $\mathrm{CdZnS} / \mathrm{ZnS}$ QDs dispersed in toluene at room temperature. The inset shows the corresponding TEM image and the schematic structure of CdZnS/ZnS alloyed-core/shell QDs. b) Temperature dependent spectrally integrated PL intensity of CdZnS/ZnS QDs. c) Temperature dependent peak energy and FWHM of PL spectra of CdZnS/ZnS QDs.

determined from the transmission electron microscopy (TEM) (Figure 1a, left inset) image, which make them fall into the weak confinement regime. ${ }^{[20,23]}$ The narrow linewidth, together with the ZnS shell acting as the spacer-layer, could effectively suppress the undesired inter-particle nonradiative energy transfer in the QDs ensembles (see detailed experimental demonstration in the Supporting Information). ${ }^{[22]}$ By virtue of the gradual composition transition from CdZnS alloyed-core into ZnS shell, ${ }^{[21,23]}$ the strain or defects in the interfaces and surfaces can be dramatically reduced, ${ }^{[20]}$ leading to a high PL quantum yield of up to $80 \%$, which is further verified by the temperature dependent PL measurements. The evolution of PL spectra and the corresponding spectrally integrated PL intensities of CdZnS/ZnS QDs as a function of temperature excited at $395 \mathrm{~nm}$ are shown in Figure $1 \mathrm{~b}$ and Figure S2. It is found that the CdZnS/ZnS QDs exhibit less than 10\% thermal quenching as the temperature changes from 10 to $300 \mathrm{~K}$, indicating the nonradiative recombination centers or defects, which are detrimental to achieving lasing, are minimal.[24,25] Similar to the common binary QDs, the PL peak wavelengths of the ternary CdZnS/ZnS QDs show redshift and the FWHM broadens with increasing temperature (Figure 1c) (see detailed discussion in the Supporting Information). ${ }^{[25,26]}$

To study the stimulated emission of the CdZnS/ZnS QDs, the prototypical self-assembled film of these QDs was investigated using a stripe pumping configuration excited at optical wavelength of $395 \mathrm{~nm} \cdot{ }^{[2,9]}$ Noticeably, the pulse-width of the excitation pulse is $5 \mathrm{~ns}$, which is one order of magnitude longer than the biexcitonic Auger recombination lifetime ( 350 ps, shown later) of the CdZnS/ZnS QDs, thus the QDs are pumped in the q-CW regime. ${ }^{[17,18]}$ It is worth mentioning that $\mathrm{q}-\mathrm{CW}$ operation is much more favorable in view of practical applications, in which the optical gain sustaining a time longer than the typically short AR lifetime is usually desired. ${ }^{[18,27]}$ On the other hand, expensive sub-picosencond pulsed lasers were extensively used as the excitation source in order to overcome the AR, ${ }^{[7,28]}$ low threshold QDs laser that is pumpable with compact Q-switched nanosecond lasers is still under exploration to reduce the cost for QDs laser technology.[27] Figure S3 presents the power dependent PL spectra from solid film of these QDs. The emergence of a narrow peak with FWHM of $\sim 5 \mathrm{~nm}$ and the nonlinear dependence of the integrated PL intensity from 435 to $445 \mathrm{~nm}$ on the pump pulse energy indicate the achievement of stimulated emission with a quite low threshold of $22 \mu \mathrm{J} /$ pulse (corresponding to $14.7 \mathrm{~mJ} / \mathrm{cm}^{2}$ ). Notably, the stimulated emission peak locates on the red side $(\sim 40 \mathrm{~nm})$ of the corresponding spontaneous emission maximum, which is consistent with the biexcitonic lasing mechanism. ${ }^{[1,29]}$ Due to the double degeneracy of the lowest emission level for II-VI type-I QDs, the population inversion can only be realized when the average number of excitons per $\mathrm{QD},\left\langle\mathrm{N}>\right.$, is larger than one. ${ }^{[1]}$ As a result, the lasing emission originates from biexciton emission, and the redshift arises from the attractive exciton-exciton interactions (positive biexciton binding energy). ${ }^{[7]}$ Our pumpprobe measurements also reveal the achievement of optical gain at $\langle\mathrm{N}\rangle=1.9$ (shown later), further confirming the biexcitonic lasing mechanism.

The challenges of realizing lasing from QDs solutions lie in the typical low loading fraction of QDs in solutions ${ }^{[9,15]}$ and the photoinduced absorption associated with the surface or interface defects. ${ }^{[7,30]}$ According to the above results, it should be highly possible to address these difficulties by employing the untraditional CdZnS/ZnS QDs as gain media because of the minimal density of defects and that the ultra-low lasing threshold from the solid films suggests the possibility of achieving lasing with a low loading factor. To explore the lasing action from these CdZnS/ZnS QDs solution, fused silica hollow fibers were employed as the resonators because the circular cross-section can naturally serve as a WGM cavity. ${ }^{[16,31,32]}$ The CdZnS/ZnS QDs solution was infiltrated into the fiber by capillary effect, and then the two ends were sealed with wax. Optical study of individual fiber was performed by employing a micro-photoluminescence ( $\mu$-PL) system (Figure 2, inset). ${ }^{[33]}$ The same laser ( $395 \mathrm{~nm}, 5 \mathrm{~ns}, 20 \mathrm{~Hz}$ ) used for the film test was employed as the pumping source. Figure 2 shows the evolution of the $\mu$-PL spectra from a fiber with an inner (outer) diameter of $\sim 54 \mu \mathrm{m}(\sim 78 \mu \mathrm{m})$ (Figure S5a) as a function of pump pulse energy. We can see that under relatively low pump energies of $<30.2 \mu \mathrm{J} / \mathrm{pulse}$, the $\mu$-PL spectra are dominated by spontaneous emission with a FWHM of $\sim 18 \mathrm{~nm}$. As the pump energy increases, sharp peaks appear and superimpose on the corresponding stimulated emission peaks, clearly revealing the 


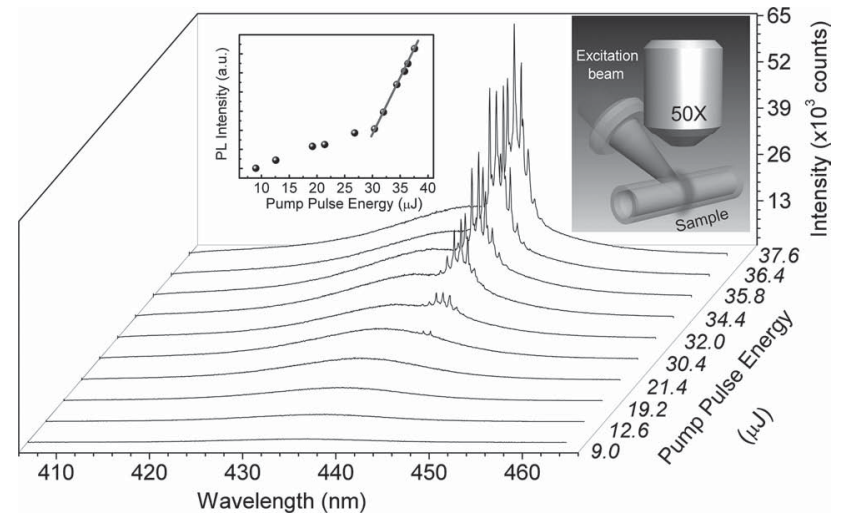

Figure 2. $\mu$-PL spectra of $\mathrm{CdZnS/ZnS} \mathrm{QDs} \mathrm{solution} \mathrm{filled} \mathrm{in} \mathrm{a} \mathrm{single}$ hollow fiber with an inner diameter of $\sim 54 \mu \mathrm{m}$ with increasing pump energy-indicating the development of lasing action with pump energy above a certain value. The left inset shows the spectrally integrated PL intensity from 435 to $445 \mathrm{~nm}$ as a function of the pump pulse energy, further confirming the achievement of lasing action with a threshold of $30.2 \mu \mathrm{J} /$ pulse. The right inset schematically depicts the pumping and collection configuration using a $\mu-P L$ system.

transition from spontaneous emission to lasing action. ${ }^{[2]}$ The corresponding optical images are presented in Figure S4. The plot of spectrally integrated PL intensity from 435 to $445 \mathrm{~nm}$ over the pump pulse energy exhibits an abrupt increase at certain points (Figure 2 inset), indicating the achievement of lasing action with such a low threshold of $30.2 \mu \mathrm{J} /$ pulse (corresponding to $\sim 25.2 \mathrm{~mJ} / \mathrm{cm}^{2}$ ). The lasing threshold is two orders of magnitude lower than that of red-emitting CdSe/ZnS QDs in solution and comparable to that of red-emitting CdSe/ZnS nanorods in solution under similar excitation conditions. ${ }^{[16]}$

It is worth noting that toluene rather than hexane was used as the solvent due to its high refractive index $(\sim 1.51$ at $\sim 450 \mathrm{~nm}),{ }^{[34]}$ ensuring the total internal reflection occurring at the interface of the inner wall as depicted in the inset of Figure $3 \mathrm{~b}$. In order to better understand the lasing mechanism, we tentatively examined the lasing peaks using WGM model. ${ }^{[33,35]}$ Considering the first radial mode order $(q=1)$, the resonant condition is given by ${ }^{[36]}: m \lambda_{m}=\pi n D$, where $m$ and $\lambda_{\mathrm{m}}$ are the angular mode number and the resonant wavelength at $m$, respectively, $n$ is the effective refractive index of the QDs solution and $D$ is the inner fiber diameter. Following the procedure reported by $\mathrm{Ta}$ et al., ${ }^{[37]}$ the lasing peaks are found to match well with the mode numbers indexed as 597-603, supporting the WGM lasing mechanism. Furthermore, the characteristics of lasing action were studied using fibers with various diameters by examining the size-dependent free spectral range $(F S R)$. As presented in Figure 3b and Figure S6-S8, the experimentally measured FSR displays a $1 / D$ dependence on the inner fiber
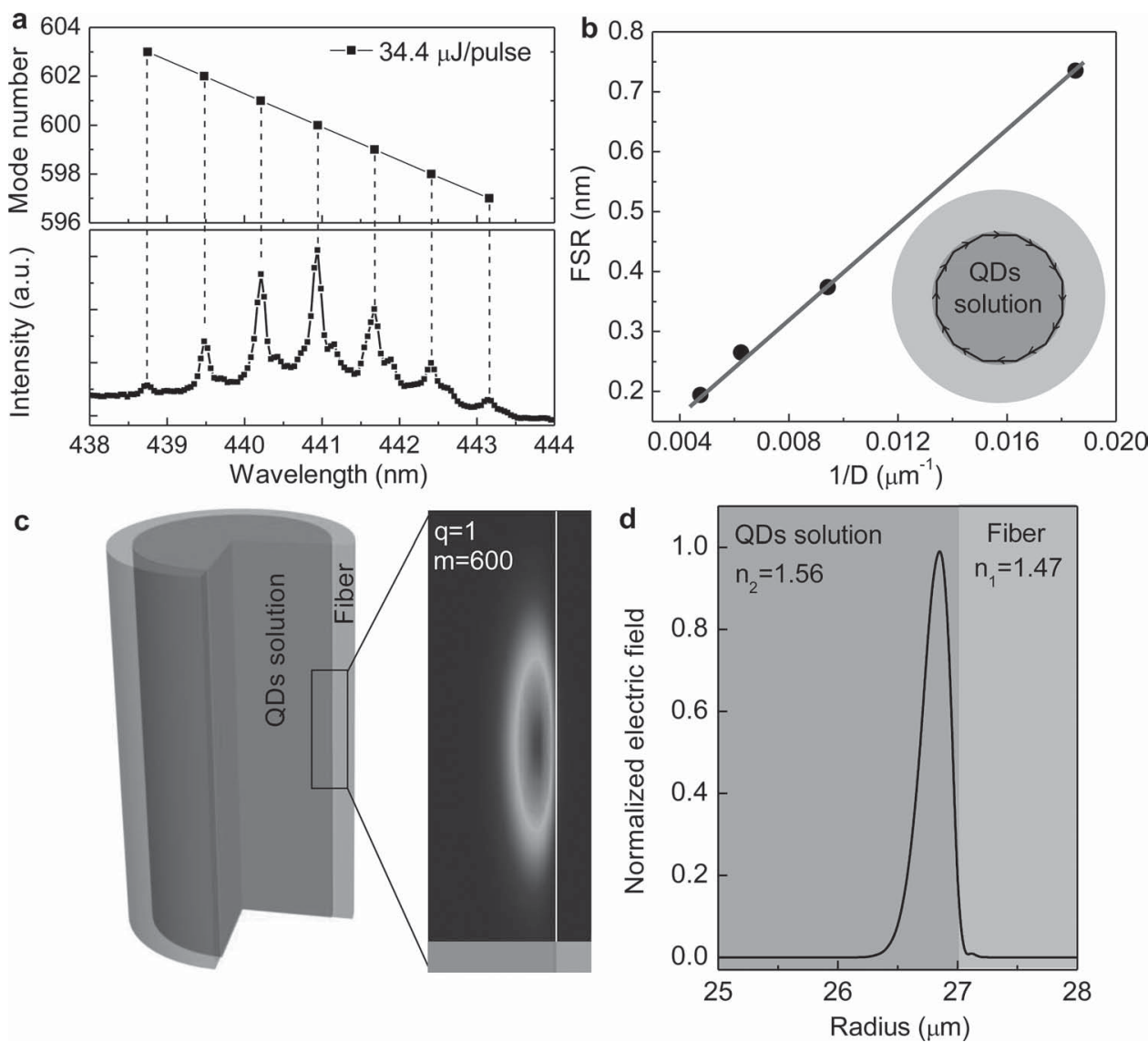

Figure 3. a) Lasing modes are well assigned according to $2 D$ WGM model, supporting WGM lasing mechanism. b) FSR is found to be inversely proportional to the inner diameter of the fibers, further confirming the WGM lasing mechanism. The inset depicts the principle of the WGM lasing. c) Electric field distribution ( $p=1, m=600$ ) for the WGM cavity in the radial direction. $d$ ) Normalized field profile ( $p=1, m=600)$ for the WGM cavity in the radial direction. 
diameter. According to the 2D WGM model, the FSR is given by ${ }^{[31]}: F S R=\lambda^{2} / \pi n D$, which means that FSR is theoretically inversely proportional to the inner fiber diameter, further corroborating the WGM lasing mechanism. Thanks to the high refractive index contrast between the solution (1.56) and the glass $(1.47$ at $440 \mathrm{~nm}),{ }^{[38]}$ the QDs emission is strongly confined inside the cavity. This is supported by numerical simulation using finite element method (FEM) available from COMSOL multiphysics as shown in Figure 3c. ${ }^{[31,35]}$ The electric field distribution in the radial direction of WGM $(p=1, m=600)$ in a $54 \mu \mathrm{m}$-diameter capillary tube exhibits effective optical confinement close to the cavity boundary. In addition, the normalized field profile across centre of the optical mode is shown in Figure 3d, we can see that there is only a small fraction of evanescent wave coupling outside the QD-glass interface. The tiny optical loss of our structure contributes to the obtained low threshold lasing, accompanied with a high lasing $Q$-factor of $\sim 3000$ (calculated by $Q=\lambda / \Delta \lambda,{ }^{[39]}$ where $\lambda$ and $\Delta \lambda$ are the lasing peak wavelength and the corresponding linewidth of the lasing peak, respectively).

Deeper insights into the observation of lasing action from CdZnS/ZnS QDs solution could be obtained through accessing the exciton dynamics by time-resolved PL measurements. Figure 4a presents the PL decays of CdZnS/ZnS QDs solution filled in 1-mm-thick cuvette recorded at the peak wavelength with varying excitation intensities (excitation wavelength: $395 \mathrm{~nm})$. Under low excitation intensities $(<\sim 85 \mu \mathrm{J} /$ $\mathrm{cm}^{2}$ ), the PL decay curves do not change with variations of excitation intensity, which is consistent with the single exciton recombination. ${ }^{[40]}$ The single exciton decay can be well-fitted by a double-exponential-decay function with $\tau_{1}=2.1 \mathrm{~ns}$ and $\tau_{2}$ $=9.9 \mathrm{~ns}$, which is common and mostly attributed to two different radiative channels of the core-state (the shorter lifetime component, $\tau_{1}=2.1 \mathrm{~ns}$ ) and the surface-state (the longer lifetime component, $\tau_{2}=9.9 \mathrm{~ns}$ ) recombination, respectively. ${ }^{[41,42]}$ As excitation intensity increases, an obvious fast decay process emerges, corresponding to the nonradiative biexcitonic AR. ${ }^{[40,43]}$ Using subtraction procedure developed by Klimov et al., ${ }^{[8]}$ the AR lifetime was deduced to be $\sim 350 \mathrm{ps}$, which is dozens of times longer than that of blue-emitting CdSe/ZnS QDs and comparable to or even larger than those of red-emitting CdSebased QDs. ${ }^{[2,8]}$ Such a long AR lifetime of CdZnS/ZnS QDs is very beneficial for achieving lasing and can be mainly attributed to two reasons: (i) as mentioned above, the CdZnS/ZnS QDs belong to the weak confinement regime; therefore, the overlap between electron and hole wave functions is not as strong as in the blue-emitting CdSe/ZnS QDs - in consequence, the AR could be mitigated; and (ii) the gradual composition transition from the core to shell results in a smooth interfacial potential, which would further suppress AR as a loose potential profile will inhibit AR by more strict momentum conservations compared to a steep interface potential existing in normal coreshell QDs. ${ }^{[21,44,45]}$ In addition, the transient dynamics of the laser device made by infiltrating CdZnS/ZnS QDs solution
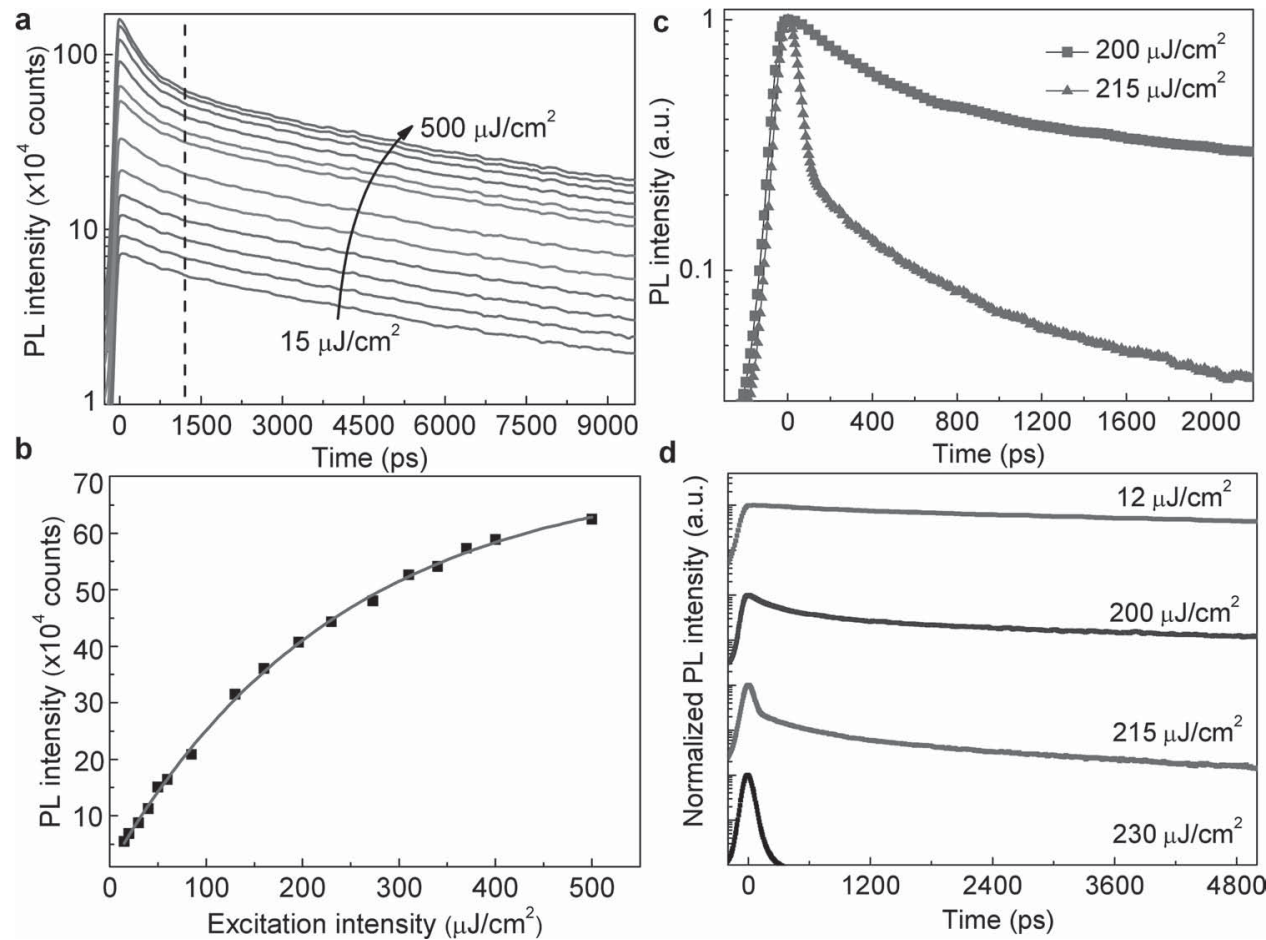

Figure 4. a) PL decays of $C d Z n S / Z n S$ QDs solution filled in a 1-mm-thick cuvette under varying excitation intensities ranging from 15 to $500 \mu \mathrm{J} / \mathrm{cm}^{2}$ excited at $395 \mathrm{~nm}$. The biexcitonic Auger lifetime of CdZnS/ZnS QDs is extracted to be $\sim 350 \mathrm{ps}$. b) PL intensities at delay time of $1.2 \mathrm{~ns}$ from CdZnS/ ZnS QDs solution filling in the 1-mm-thick cuvette under varying excitation intensity levels. The solid line represents the best fitting curve based on the Poisson distribution of the number of excitons following excitation. The concentration of CdZnS/ZnS QDs solution is determined to be $\sim 2.0 \times 10^{-5} \mathrm{~mol} / \mathrm{L}$. c) Time-resolved PL traces of the laser device made by infiltrating $\mathrm{CdZnS} / \mathrm{ZnS}$ QDs solution into the fiber just below $\left(200 \mu \mathrm{J} / \mathrm{cm}^{2}\right)$ (solid square) and above $\left(215 \mu \mathrm{J} / \mathrm{cm}^{2}\right.$ ) (solid triangle) the lasing threshold $\left(210 \mu \mathrm{J} / \mathrm{cm}^{2}\right.$ - (wavelength: $395 \mathrm{~nm}$, pulse-width: $100 \mathrm{fs}$, repetition rate: $1000 \mathrm{~Hz}$ ). d) Evolution of PL dynamics as the excitation intensities vary from far below lasing threshold to far above the lasing threshold. 
into the fiber was also studied. Figure 4c displays the decay curves monitored at $440 \mathrm{~nm}$ for excitation intensities just below $\left(200 \mu \mathrm{J} / \mathrm{cm}^{2}\right)$ the lasing threshold $\left(210 \mu \mathrm{J} / \mathrm{cm}^{2}\right)$, where AR already occurs, and just above $\left(215 \mu \mathrm{J} / \mathrm{cm}^{2}\right)$ the threshold pumped by a femtosecond amplified-pulsed laser (wavelength: $395 \mathrm{~nm}$, pulse-width: $100 \mathrm{fs}$, repetition rate: $1000 \mathrm{~Hz}$ ). We can come to the conclusion that although the nonradiative AR is rapid compared to spontaneous emission so as to effectively deplete the excited carrier population, the development of lasing action, whose timescale is measured to be less than 50 ps (limited by time resolution of our streak camera system), is fast enough to compete against AR. The evolution of PL dynamics under different excitation intensities can be clearly seen in Figure 4d. At a low excitation intensity of far below threshold $\left(12 \mu \mathrm{J} / \mathrm{cm}^{2}\right)$, the emission follows the slow single exciton decay. Upon increasing the excitation intensity approaching the threshold $\left(200 \mu \mathrm{J} / \mathrm{cm}^{2}\right)$, the rapid AR manifests. As the excitation intensity further increases to just over the threshold $\left(215 \mu \mathrm{J} / \mathrm{cm}^{2}\right)$, an even faster decay channel, corresponding the lasing action, emerges. Finally, the emission totally collapes to $<50$ ps when the excitation intensity far exceeds the threshold $\left(230 \mu \mathrm{J} / \mathrm{cm}^{2}\right)$, indicating the dominance in the decay process by lasing action. Importantly, on the basis of the fast AR as compared to single exciton recombination, all the excited CdZnS/ZnS QDs will decay into single exciton state at long enough decay times. Consequently, the PL intensity at decay time of $1.2 \mathrm{~ns}$ as depicted by the vertical dashed line in Figure $4 \mathrm{a}$ is proportional to $1-P_{0}$, where $P_{0}$ is the probability of non-excited QDs in the ensemble ${ }^{[40]}$ and is described by: $P_{0}=e^{-N}$, where $<N>$ is the average number of excitons per QD following photoexcitation. By fitting the excitation intensity dependent PL signal at $1.2 \mathrm{~ns}$ (Figure 4b) extracted from Figure $4 \mathrm{a}$, the absorption cross-section of CdZnS/ZnS QDs is derived to be $\sim 2.1 \times 10^{-15} \mathrm{~cm}^{2}$ at $395 \mathrm{~nm}$. Therefore, the concentration of CdZnS/ZnS QDs solution is determined to be $\sim 2.0 \times 10^{-5} \mathrm{~mol} / \mathrm{L}$. Such low concentration of $\mathrm{CdZnS} / \mathrm{ZnS}$ QDs results in an effective loading factor of only $0.079 \%$ considering the CdZnS-core size of $\sim 5 \mathrm{~nm}$, which is much lower than the predicted minimum value $(0.2 \%)$ to achieve stimulated emission or lasing for CdSe-based QDs. ${ }^{[1]}$ This discrepancy may stem from the intrinsic gain property of CdZnS/ZnS QDs differing from that of CdSe/ZnS QDs. ${ }^{[15]}$ To this end, pump-probe measurements were carried out to derive the gain cross-section of CdZnS/ZnS QDs. In these experiments, the time evolution of the transient absorption $(\Delta \alpha)$ is monitored by changing the delay between pump and probe beam (Figure 5b inset). ${ }^{[46]}$ Optical gain manifests as $-\Delta \alpha / \alpha_{0}>1$, where $\alpha_{0}$ is the linear absorbance at probe wavelength. ${ }^{[28]}$ Figure 5 a presents the time evolution of $-\Delta \alpha / \alpha_{0}$ of $\mathrm{CdZnS} / \mathrm{ZnS}$ QDs solution under various excitation intensities at the pump and probe wavelengths of 400 and $440 \mathrm{~nm}$ (in resonance with the lasing peaks), respectively. The optical gain can be clearly observed when excitation intensity is above $0.52 \mathrm{~mJ} / \mathrm{cm}^{2}$, corresponding to $\left.<\mathrm{N}\right\rangle=\sim 1.9$, and could sustain as long as $\sim 50$ ps under pumping intensity of $1.4 \mathrm{~mJ} / \mathrm{cm}^{2}$. In contrast, when the probe wavelength moves away from the wavelength range of the lasing peaks, the maximal transient absorption signal, $-\Delta \alpha / \alpha_{0}$, will saturate below 1 as depicted in Figure 5b. Based on these results, the maximum net gain of CdZnS/ZnS QDs solution was deduced to be 0.53 $\mathrm{cm}^{-1}$ by using the relation ${ }^{[46]}: I_{P}=I_{0} e^{g d}$, where $I_{\mathrm{p}}$ and $I_{0}$ are the
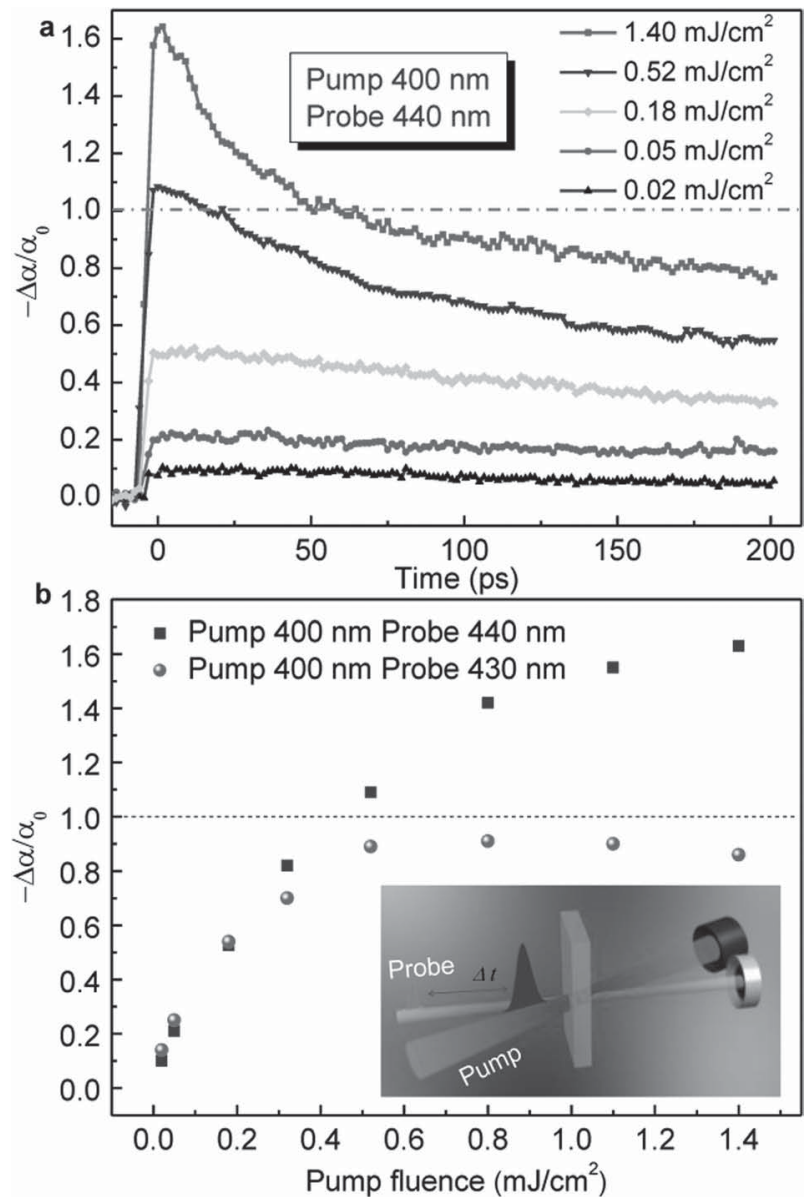

Figure 5. a) Dynamics of transient absorption changes normalized by the ground-state absorption $\left(-\Delta \alpha / \alpha_{0}\right)$ at $440 \mathrm{~nm}$ with increasing pump intensity. Gain can be clearly observed with excitation intensities above $0.52 \mathrm{~mJ} / \mathrm{cm}^{2}$ and can sustain $\sim 50 \mathrm{ps}$ at excitation intensity of $1.40 \mathrm{~mJ} / \mathrm{cm}^{2}$. b) Transient absorption changes normalized by the ground-state absorption $\left(-\Delta \alpha / \alpha_{0}\right)$ at $440 \mathrm{~nm}$ and $430 \mathrm{~nm}$ as a function of excitation intensity. The gain cross-section of CdZnS/ZnS QD is estimated to be $5 \times 10-{ }^{17} \mathrm{~cm}^{2}$. The inset portrays the principle of pump-probe measurements.

transmitted probe beam intensity in presence of pump beam and the probe beam intensity before passing the sample, $g$ is the net gain and $d$ is the thickness of the sample. Therefore, the gain cross-section per CdZnS/ZnS QD is estimated to be $5 \times 10^{-17} \mathrm{~cm}^{2}$, which is $\sim 3$-times larger than that of 2.6-nm sized CdSe QDs used for the minimum loading factor calculation, hence justifying the observation of lasing action from the dilute CdZnS/ZnS QDs solution below the predicted minimum volume fraction.

In conclusion, for the first time, we have demonstrated blue liquid WGM lasing from solution of ternary CdZnS/ZnS alloyed-core/shell QDs. The low density of defects, suppressed $\mathrm{AR}$, and large gain cross-section of CdZnS/ZnS QDs as well as the exploitation of high $Q$-factor WGM resonators enable us to observe lasing action from dilute CdZnS/ZnS QDs solution even below the predicted minimum loading fraction for CdSe-based QDs with such low thresholds that are pumpable in q-CW regime using a compact nanosecond laser. These findings could offer new possibilities in blue laser related areas and 
push ahead toward full-color visible lasers based on colloidal QDs and may find applications in biophotonics including optofluidic lasers and sensors.

\section{Experimental Section}

Synthesis of Ternary CdZnS/ZnS Alloyed-Core/Shell QDs: Typically, cadmium oxide (CdO, $1 \mathrm{mmol})$, zinc acetate $\left(\mathrm{Zn}(\text { acet })_{2}, 10 \mathrm{mmol}\right)$, and $\mathrm{OA}(7 \mathrm{mmol})$ were mixed in a $100 \mathrm{~mL}$ flask. The mixture was evacuated for $20 \mathrm{~min}$ and heated up to $150{ }^{\circ} \mathrm{C}$ under nitrogen condition. Then, $15 \mathrm{~mL}$ 1-octadecene (1-ODE) was added into the mixture and the temperature was further increased to $300{ }^{\circ} \mathrm{C}$. Following that, the sulfur precursor obtained by dissolving $\mathrm{S}$ powder $(2 \mathrm{mmol})$ into $3 \mathrm{~mL} 1-\mathrm{ODE}$ was quickly injected into the mixture. After $8 \mathrm{~min}$ for the reaction at a constant temperature of $300{ }^{\circ} \mathrm{C}, 8 \mathrm{mmol} \mathrm{S}$ powder dissolved in TBP (TPBS) was added into the mixture. The mixture was cooled down to room temperature after the reaction ended. Finally, the QDs were purified and dissolved in toluene for further experiments.

Steady State and Ultrafast Dynamics Studies: For the temperature dependent PL measurements, the CdZnS/ZnS QDs were dispersed in poly (methyl methacrylate) (PMMA) in order to avoid the possible influence of interdot Förster resonance energy transfer, and then were spin-coated onto a sapphire substrate. The samples were mounted in a helium closed-cycle cryostat and excited at $395 \mathrm{~nm}$ provided by a Xenon lamp equipped with a monochromator. The excitation intensity was kept low to avoid any extra irradiation effect. For the $\mu$-PL measurements, the laser beam (wavelength: $395 \mathrm{~nm}$, pulse-width: $5 \mathrm{~ns}$, repetition rate: $20 \mathrm{~Hz}$ ) from a wavelength-tunable nanosecond laser system was directed and focused to an elliptical spot of $-300 \times 400 \mu \mathrm{m}^{2}$. The PL signal was collected through a microscope objective $(50 \times, N A=0.42)$. Part of the $\mathrm{PL}$ signal was dispersed by a $750 \mathrm{~mm}$ monochromator and detected by a silicon-charged coupled device (CCD), while the remanent part was sent to a camera for imaging. Suitable filters were used to block the residual excitation light. For the time resolved PL measurements, the CdZnS/ $\mathrm{ZnS}$ QDs solution in a 1-mm-thick quartz cuvette was excited at $395 \mathrm{~nm}$ (pulse-width: $100 \mathrm{fs}$, repetition rate: $1000 \mathrm{~Hz}$ ) with a wavelength-tunable femtosecond amplified laser system. The PL signals were recorded by an Optronics streak camera with a temporal resolution of $\sim 50 \mathrm{ps}$. For the pump-probe measurements, the same femtosecond laser system was employed. The CdZnS/ZnS QDs solution in a 1-mm-thick quartz cuvette was pumped at $400 \mathrm{~nm}$ (beam size: $\sim 1 \mathrm{~mm}$ in diameter) by frequencydoubling of the fundamental wavelength $(800 \mathrm{~nm})$ from the Ti:sapphire regenerative amplifier. The probe beam (beam size: $\sim 0.5 \mathrm{~mm}$ ), provided by an optical parameter amplifier operating at a wavelength tunable from 260 to $2600 \mathrm{~nm}$, was detected by a Si photodiode using standard lock-in amplifier technique.

\section{Supporting Information}

Supporting Informtion is available from the Wiley Online Library or from the author.

\section{Acknowledgements}

This work is supported by the Singapore National Research Foundation through the Competitive Research Programme (CRP) under Project No. NRF-CRP6-2010-02 and the Singapore Ministry of Education through the Academic Research Fund under Project MOE2011-T3-1-005 (Tier 3). H.V.D. also gratefully acknowledges the support from NRF-RF-2009, ESF-EURYI and TUBA.

Received: July 18, 2014

Revised: August 26, 2014

Published online: September 18, 2014
[1] V. I. Klimov, A. A. Mikhailovsky, S. Xu, A. Malko, J. A. Hollingsworth, C. A. Leatherdale, H. J. Eisler, M. G. Bawendi, Science 2000, 290, 314.

[2] Y. Wang, V. D. Ta, Y. Gao, T. C. He, R. Chen, E. Mutlugun, H. V. Demir, H. D. Sun, Adv. Mater. 2014, 26, 2954.

[3] Q. Sun, Y. A. Wang, L. S. Li, D. Wang, T. Zhu, J. Xu, C. Yang, Y. Li, Nature Photon. 2007, 1, 717.

[4] J. Kwak, W. K. Bae, D. Lee, I. Park, J. Lim, M. Park, H. Cho, H. Woo, D. Y. Yoon, K. Char, S. Lee, C. Lee, Nano Lett. 2012, 12, 2362.

[5] H. J. Eisler, V. C. Sundar, M. G. Bawendi, M. Walsh, H. I. Smith, V. Klimov, Appl. Phys. Lett. 2002, 80, 4614.

[6] T. R. G. W. P. Risk, A. V. Nurmikko, Compact Blue-green Lasers, Cambridge Univ. Press, 2003.

[7] V. I. Klimov, Annu. Rev. Phys. Chem. 2007, 58, 635.

[8] V. I. Klimov, A. A. Mikhailovsky, D. W. McBranch, C. A. Leatherdale, M. G. Bawendi, Science 2000, 287, 1011.

[9] C. Dang, J. Lee, C. Breen, J. S. Steckel, S. Coe-Sullivan, A. Nurmikko, Nature Nanotechnol. 2012, 7, 335.

[10] M. Kazes, T. Saraidarov, R. Reisfeld, U. Banin, Adv. Mater. 2009, 21, 1716.

[11] C. Grivas, C. Li, P. Andreakou, P. Wang, M. Ding, G. Brambilla, L. Manna, P. Lagoudakis, Nature Comm. 2013, 4, 2376.

[12] Q. Darugar, W. Qian, M. A. El-Sayed, Appl. Phys. Lett. 2006, 88, 261108.

[13] Y. Chen, L. Lei, K. Zhang, J. Shi, L. Wang, H. Li, X. M. Zhang, Y. Wang, H. L. W. Chan, Biomicrofluidics 2010, 4, 043002.

[14] H. Schmidt, A. R. Hawkins, Nature Photon. 2011, 5, 598.

[15] J. Schaefer, J. P. Mondia, R. Sharma, Z. H. Lu, A. S. Susha, A. L. Rogach, L. J. Wang, Nano Lett. 2008, 8, 1709.

[16] M. Kazes, D. Y. Lewis, Y. Ebenstein, T. Mokari, U. Banin, Adv. Mater. 2002, 14, 317

[17] D. Oron, M. Kazes, U. Banin, Phys. Rev. B 2007, 75, 035330.

[18] D. Oron, M. Kazes, I. Shweky, U. Banin, Phys. Rev. B 2006, 74, 115333.

[19] R. Chen, D. Li, B. Liu, Z. Peng, G. G. Gurzadyan, Q. Xiong, H. Sun, Nano Lett. 2010, 10, 4956.

[20] W. K. Bae, M. K. Nam, K. Char, S. Lee, Chem. Mater. 2008, 20, 5307.

[21] X. Wang, X. Ren, K. Kahen, M. A. Hahn, M. Rajeswaran, S. Maccagnano-Zacher, J. Silcox, G. E. Cragg, A. L. Efros, T. D. Krauss, Nature 2009, 459, 686.

[22] K. H. Lee, J. H. Lee, W. S. Song, H. Ko, C. Lee, J. H. Lee, H. Yang, ACS Nano 2013, 7, 7295.

[23] X. H. Zhong, Y. Y. Feng, W. Knoll, M. Y. Han, J. Am. Chem. Soc. 2003, 125, 13559

[24] G. Raino, T. Stoeferle, I. Moreels, R. Gomes, J. S. Kamal, Z. Hens, R. F. Mahrt, ACS Nano 2011, 5, 4031.

[25] P. Jing, J. Zheng, M. Ikezawa, X. Liu, S. Lv, X. Kong, J. Zhao, Y. Masumoto, J. Phys. Chem. C 2009, 113, 13545.

[26] D. Valerini, A. Creti, M. Lomascolo, L. Manna, R. Cingolani, M. Anni, Phys. Rev. B 2005, 71, 235409.

[27] C. Dang, J. Lee, K. Roh, H. Kim, S. Ahn, H. Jeon, C. Breen, J. S. Steckel, S. Coe-Sullivan, A. Nurmikko, Appl. Phys. Lett. 2013, 103,171104

[28] A. A. Mikhailovsky, A. V. Malko, J. A. Hollingsworth, M. G. Bawendi, V. I. Klimov, Appl. Phys. Lett. 2002, 80, 2380.

[29] M. Saba, S. Minniberger, F. Quochi, J. Roither, M. Marceddu, A. Gocalinska, M. V. Kovalenko, D. V. Talapin, W. Heiss, A. Mura, G. Bongiovanni, Adv. Mater. 2009, 21, 4942.

[30] A. V. Malko, A. A. Mikhailovsky, M. A. Petruska, J. A. Hollingsworth, V. I. Klimov, J. Phys. Chem. B 2004, 108, 5250.

[31] V. D. Ta, R. Chen, L. Ma, Y. J. Ying, H. D. Sun, Laser Photonics Rev. 2013, 7, 133

[32] L. He, S. K. Oezdemir, J. Zhu, W. Kim, L. Yang, Nature Nanotechnol. 2011, 6, 428.

[33] R. Chen, B. Ling, X. W. Sun, H. D. Sun, Adv. Mater. 2011, 23, 2199. 
[34] M. P. K. Moutzouris, S. C. Betsis, I. Stavrakas, G. Hloupis, D. Triantis, Appl. Phys. B 2013, 116, 617.

[35] M. Oxborrow, IEEE Trans. Microwave Theory Tech. 2007, 55, 1209.

[36] H. H. Fang, R. Ding, S. Y. Lu, Y. D. Yang, Q. D. Chen, J. Feng, Y. Z. Huang, H. B. Sun, Laser Photonics Rev. 2013, 7, 281

[37] V. D. Ta, R. Chen, H. D. Sun, Adv. Mater. 2012, 24, OP60.

[38] W. F. Zhang, H. Zhu, S. F. Yu, H. Y. Yang, Adv. Mater. 2012, 24, 2263.

[39] R. Chen, T. Van Duong, H. D. Sun, Sci. Rep. 2013, 3, 244.

[40] S. A. Ivanov, M. Achermann, ACS Nano 2010, 4, 5994.
[41] X. Y. Wang, L. H. Qu, J. Y. Zhang, X. G. Peng, M. Xiao, Nano Lett. 2003, 3, 1103

[42] Z. Gu, L. Zou, Z. Fang, W. Zhu, X. Zhong, Nanotechnology 2008, 19, 135604.

[43] H. Htoon, J. A. Hollingsworth, R. Dickerson, V. I. Klimov, Phys. Rev. Lett. 2003, 91, 227401.

[44] F. Garcia-Santamaria, Y. F. Chen, J. Vela, R. D. Schaller, J. A. Hollingsworth, V. I. Klimov, Nano Lett. 2009, 9, 3482.

[45] Y. S. Park, W. K. Bae, L. A. Padilha, J. M. Pietryga, V. I. Klimov, Nano Lett. 2014, 14, 396.

[46] L. Pavesi, L. Dal Negro, C. Mazzoleni, G. Franzo, F. Priolo, Nature $2000,408,440$ 